\title{
LEER Y ESCRIBIR: ASUNTOS DISFRUTABLES
}

\author{
Sylvia Doyenart y Gladys Pagano
}

\section{INTRODUCCIÓN}

A fines del siglo XX, los niños son atraídos por diferentes medios de comunicación y avances tecnológicos; es difícil encontrar niños que elijan un libro en su tiempo de esparcimiento. Si rescatamos el comentario de docentes en particular y de la comunidad en general coinciden en que el niño de hoy no lee. La lectura se identifica con una actividad "por obligación", poco gratificante.

En el estudio realizado por Equipos Consultores en 1988 "El público lector y los usuarios de la Biblioteca" se desprende que 7 de cada 10 uruguayos nunca consultaron una Biblioteca y el 49,6\% de los montevideanos no lee libros de ningún tipo. El informe de la CEPAL (1990-91) expresa que aproximadamente en 1 de cada 5 hogares uruguayos no hay un solo libro.

¿Qué sucede en nuestro contexto laboral? Nuestra tarea docente se lleva a cabo en un Centro Educativo judío, de doble turno, donde la enseñanza se imparte en tres idiomas y donde se realizan actividades extracurriculares libres y optativas. Se obtienen excelentes niveles de rendimiento en todas las áreas, que se ven reflejados no sólo en el Centro Educativo sino también en pruebas y competencias nacionales e internacionales. Como lo demuestran, entre otros, los concursos de cuentos, que han logrado premios en competencias iberoamericanas, y las pruebas nacionales aplicadas en 1996 a todos los sextos años del país por la Unidad de Medición de Resultados Educativos (U.M.R.E.).

\section{Evaluación de sextos años a nivel nacional de la U.M.R.E. 1996}

\begin{tabular}{|l|c|c|c|c|}
\hline $\begin{array}{l}\text { Porcentaje de alumnos } \\
\text { suficientes en: }\end{array}$ & $\begin{array}{c}\text { Centro } \\
\text { Educativo } \\
\text { Judío }\end{array}$ & $\begin{array}{c}\text { Escuelas de } \\
\text { contexto social } \\
\text { muy favorable } \\
\text { del dpto. }\end{array}$ & $\begin{array}{c}\text { Escuelas de } \\
\text { contexto social } \\
\text { muy favorable } \\
\text { del país }\end{array}$ & Total país \\
\hline Lengua materna & $\mathbf{9 8 . 1}$ & 88.1 & 85.2 & 57.1 \\
\hline Comprensión de texto argumentativo & $\mathbf{9 4 . 4}$ & 82.2 & 80.2 & 57.0 \\
\hline Comprensión de texto narrativo & $\mathbf{9 6 . 3}$ & 86.2 & 83.9 & 64.5 \\
\hline Reflexiones sobre el lenguaje & $\mathbf{9 6 . 3}$ & 77.1 & 74.1 & 42.4 \\
\hline
\end{tabular}


Resultados de la E.H.I.U. en lengua materna con relación a las escuelas de la misma categoría del Departamento y del País.

\begin{tabular}{|l|c|c|c|c|}
\hline & $\begin{array}{c}\text { Centro Educativo } \\
\text { Judío }\end{array}$ & $\begin{array}{c}\text { Escuelas privadas } \\
\text { del dpto. }\end{array}$ & $\begin{array}{c}\text { Escuelas privadas } \\
\text { del país }\end{array}$ & Total país \\
\hline \% Alumnos 20-24 pts. & $\mathbf{8 1 . 1}$ & 37.6 & 33.9 & 15.8 \\
\hline \% Alumnos 14-19 pts. & $\mathbf{1 7 . 0}$ & 44.6 & 45.1 & 41.3 \\
\hline \% Alumnos 7-13 pts. & $\mathbf{1 . 9}$ & 17.1 & 19.7 & 37.7 \\
\hline \% Alumnos 0-6 pts. & $\mathbf{0 . 0}$ & 0.8 & 1.2 & 5.2 \\
\hline TOTAL & $\mathbf{1 0 0 . 0}$ & 100.0 & 100.0 & 100.0 \\
\hline
\end{tabular}

No cabe duda que un reto que a toda institución educativa se le plantea, es el de generar en sus alumnos esa voluntad de accionar el libro: hacer que leer y escribir responda a un deseo más que a una obligación.

Educadores con experiencia en el trabajo de aula de distintos medios, hemos aprendido y desaprendido mucho. La inquietud de perfeccionamiento nos llevó a realizar una investigación que nos brinda la posibilidad de explicar las motivaciones que llevan a los niños de este Centro Educativo a optar por leer y escribir frente a otras actividades lúdicas o recreativas.

Estos niños eligen, durante horas de recreo, ir a la biblioteca a "alquilar" un libro antes que salir al patio a jugar. Frente a tareas de investigación (expociencia, temas emergentes, etc.), recurren por iniciativa propia a buscar material en Internet, Biblioteca, etc. Encuestas hechas en Biblioteca dan un crecimiento sensible en el préstamo de libros. Verificamos que en efecto los libros se leen y son recomendados entre compañeros. Considerando que el niño construye el conocimiento desde un contexto social determinado, y que dicha construcción es activa, no podemos dejar de lado la influencia que tiene el medio en el que se desenvuelve. El niño aprende su lengua materna en el contexto de un marco social en el cual el lenguaje se usa de una manera funcional

Ante esto, vemos importante examinar qué estrategias son usadas a nivel docente-institucional, en nuestro Centro Educativo para estimular la lectura y la escritura en el nivel primario, siendo éste el centro de interés de nuestra investigación.

\section{OBJETIVOS}

\section{Objetivo General}

Conocer cómo y con qué alcance el Centro Educativo se constituye en un ámbito propicio para el fomento de la lectura y la escritura.

\section{Objetivos específicos}

Determinar las estrategias institucionales docentes que desarrolla el Centro Educativo en el nivel primario, en relación con la promoción de la lectura y la escritura.

Valorar con qué alcance se articulan los resultados obtenidos en nivel primario con las expectativas y objetivos abordados en educación media en lectura y escritura. 


\section{MARCO TEÓRICO}

\subsection{La educación en la sociedad actual}

La magnitud, diversidad y velocidad de los cambios que afectan a la sociedad contemporánea, imponen desafíos de todo tipo.

La educación no queda al margen, se ve la necesidad de iniciar una nueva etapa que permita fortalecer las capacidades individuales, resolver problemas, analizar críticamente la realidad que nos circunda, adecuándose al mundo y a los cambios de una manera positiva y humanitaria.

Hace ya 50 años, la Conferencia General de la UNESCO celebrada en México en 1947 se propuso como uno sus objetivos primordiales, "llevar los elementos de la cultura a sectores de población que viven al margen del mundo civilizado, por carecer de los instrumentos esenciales de cultura (lectura y escritura)". Esta declaración, parte del supuesto de que sin esos instrumentos esenciales, la vida moderna, por su complejidad, se hace inasequible, trátase pues de una educación mínima, básica, que comprende todos los elementos indispensables que posibilitan una participación decorosa en la vida actual.

Hoy, al igual que hace 50 años, educar es mucho más que enseñar a leer y a escribir más o menos eficientemente, es ofrecer la posibilidad de ejercer el derecho a integrarse plenamente a la vida contemporánea, de ser consciente y reflexivo, capaz de analizar, discutir, aceptar o no, lo que el mundo nos ofrece o intenta a veces imponernos.

En la actualidad, y particularmente en los países de la región americana, el tema de la educación ha venido ganando espacio inédito como parte de los grandes debates nacionales de las políticas públicas. En ocasión de celebrarse la XXIV sesión de la Comisión Económica para América Latina (Santiago, abril, 1992) coincidieron en asignar a la educación un papel central en las nuevas estrategias de desarrollo. Sostuvieron que la educación será la perspectiva del crecimiento económico, en el cual la incorporación de conocimientos en el proceso productivo es el eje de su crecimiento. (Pozner, 1997)

El avance tecnológico transforma al hombre en un individuo sin barreras en el espacio y el tiempo; su entorno ha cambiado. Se podría afirmar que la cultura de masas se universalizó y esto implica un cambio de mentalidad en la educación, que lleva a variar, cambiar o transformar los modelos educativos. La educación debe desarrollarse al mismo ritmo de la vida. Autores como Piaget apoyan este proceso, fortalecen esta idea viendo al aprendizaje no como un proceso en el cual el sujeto se limita a recepcionar y registrar lo que automáticamente recibe, sino como una construcción completa donde lo que es recibido del objeto y lo que es acotado por el sujeto, está indisolublemente unido. (Nicoletti, 1993).

\subsection{Estrategias de intervención}

El término educación, lo mismo que otros usados con demasiada frecuencia, han ido perdiendo su significación precisa. Si se recorren las distintas definiciones que de educación se han ido dando a lo largo del tiempo, advertimos en ellas que la idea expresada con mayor frecuencia es la de perfección, o sea que la educación aspira de manera inmediata a potenciar las facultades del hombre y, a través de ellas, a resaltar la persona humana.

Se necesitan pues educadores reflexivos, pensativos e inventivos que analicen su trabajo para considerar cómo mejorar el aprendizaje. Tener la capacidad de conocer el propio conocimiento, de pensar y reflexionar sobre cómo reaccionaremos o hemos reaccionado ante un problema o una tarea (metacognición). 
La institución de enseñanza desarrolla, implementa, recursos y estrategias para llevar a cabo ese proceso educativo que le corresponde impulsar de la manera más óptima posible. Las estrategias educativas serán pues aquellos recursos afectivos, sicomotores, cognitivos, que se llevarán a cabo para el cumplimiento eficaz de los objetivos propuestos. Serán los procesos que sirvan de base a la realización de las tareas intelectuales de asimilación y acomodación al mundo que rodea al alumno, siendo éste capaz de examinar la nueva situación, revisar su experiencia y reorganizar sus conocimientos.

Las estrategias educativas serán capaces de formar un ambiente lleno de estímulos, donde el alumno genere nuevos conocimientos a partir de su propio hacer. Estas estrategias podrán ser institucionales, creando así una cultura propia y un aprendizaje institucional, o podrán ser del aula específicamente. Estarán orientadas a facilitar y dirigir ese proceso de aprendizaje integradas a él, con la finalidad de optimizarlo. Son caminos para enfrentar y utilizar la información y es necesario que tengan coherencia institucional para que se desarrollen ámbitos generadores de aprendizaje, que sean estimuladores de esos procesos.

Las estrategias serán los caminos que le brindarán a los niños la oportunidad para que puedan llegar a descubrir y encontrarse con ambientes que los inviten a construir su propio conocimiento. Serán ellos los constructores de su propio aprendizaje, adaptándose y asimilando, el docente será el intermediario de esa situación.Tratar de discernir las estrategias generales, distinguiéndolas de las habilidades, reforzando su uso, señalando como cambian en función de los fines del conocimiento y del contexto. Fomentar en los alumnos la investigación y la motivación que les permita explorar y utilizar plenamente la capacidad de reflexionar críticamente y responder con flexibilidad a diferentes situaciones.

\subsection{Leer y escribir}

El avance tecnológico ha modificado totalmente las formas de aprehensión del código escrito, ha introducido códigos nuevos, que la propia tecnología impone. La iniciación de un niño en la lectura y la escritura no es exclusivamente entonces, una cuestión de educación formal. La interacción del ser humano con su medio está mediatizada por la cultura desde el momento mismo del nacimiento, siendo los padres, los educadores, los adultos y, en general los otros seres humanos, los principales agentes mediadores.

No debemos olvidar que leer y escribir son actividades esencialmente sociales y de acuerdo con esta postura las estrategias didácticas que se utilicen en educación deberán ser coherentes entre sí, teniendo en cuenta las relaciones interpersonales para que el Centro Educativo sea un lugar agradable "donde todos quieran estar".

Smith (1994) argumenta que los chicos realmente aprenden a leer y a escribir si son admitidos en una comunidad de usuarios del lenguaje escrito, tal como son admitidos desde su nacimiento en la comunidad de hablantes de su lengua. Una de las comunidades más importante a la que puede sumarse un individuo es la "comunidad de los alfabetizados", porque su ingreso asegura a los individuos que aprenderán a leer y a escribir, y porque la escritura es la puerta de ingreso a muchas otras comunidades.

Aprendemos a hablar, dice Halliday (1982), porque queremos hacer cosas que no podríamos hacer de otro modo, y por la misma razón aprendemos a leer y escribir. El aprendizaje es creativo, inseparable de la experiencia. La enseñanza tiene que facilitar las actividades a partir de las cuales se pueda producir la creatividad. La lectura y la escritura son empresas creativas, no transferencias de información.

48 - Universidad ORT Uruguay 
La lectura es un instrumento sin el cual es imposible acceder a los avances científicos y tecnológicos. A través de ella se fortalece la memoria colectiva y se facilitan procesos de acumulación en el arduo camino de la investigación y el conocimiento. Permite penetrar en el pensamiento del otro, independientemente de sus coordenadas témporo-espaciales y aportar al texto su propia experiencia. El placer de leer está vinculado a la forma en cómo se inicia el aprendizaje de la lectura, que va más allá de la simple descodificación de signos para convertirse en una interpretación, un diálogo entre el lector y la obra.

Se deduce pues que la lectura, al igual que la escritura, surgen del deseo o la necesidad de hacerlas, no de una imposición, sino de una necesidad propia del individuo. Enseñar sería aceptar la libertad para que el alumno pueda expresar sus vivencias, sus ideas, sus sentimientos, sus opiniones, brindar tiempo para la reflexión, la discusión de sus propios escritos.

Según Pozner (1997) la unidad educativa es el espacio indispensable para concretar aprendizajes de calidad para todos los alumnos. Es el espacio para recuperar el sentido y la significación de las prácticas pedagógicas. Para esto es esencial una buena gestión escolar, donde se involucre todo el personal: directivos, docentes, familia, alumnos. Es esencial también que se adecue al contexto, a las particularidades y necesidades propias del Centro.

La misma autora define la gestión escolar como el conjunto de acciones, relacionadas entre sí, que emprende el Equipo Directivo de un centro para promover y posibilitar la consecución de la intencionalidad pedagógica en-con-para la comunidad educativa.

El objetivo primordial de la gestión escolar sería centrar-focalizar-nuclear a la unidad educativa alrededor de los aprendizajes de los niños y jóvenes. No obstante se prioriza el involucramiento de los docentes y de la comunidad toda, para hacer factibles y alcanzables esos objetivos educativos. Deberá ser consciente y conocer muy bien el ámbito social al que va dirigida, partiendo así de un dominio social y colectivo que le da contundencia para lograr una positiva transformación de los seres humanos.

\section{DISEÑO METODOLÓGICO}

El enfoque fue predominantemente cualitativo, sin descuidar posibles abordajes cuantitativos. El método de investigación usado tiene en cuenta las exigencias de la realidad a la que se enfrenta. En este caso se combinaron los atributos naturalista y holista del paradigma cualitativo, con los atributos confirmatorios del paradigma cuantitativo.

Como lo explica Pourtois y Desmet (1992) la triangulación de las fuentes es susceptible de proporcionar nuevas informaciones a la investigación, en la medida en que hace referencia a informadores múltiples para comprender mejor las intencionalidades de los actores puestos en escena.

\subsection{Campo de investigación}

Esta investigación se llevó a cabo en un Centro Educativo específico que tiene como características esenciales el de ser una institución privada, judía, con doble turno, trilingüe (español, hebreo e inglés), cada área asistida por docentes formados para tal efecto. Los alumnos cumplen doble horario escolar con asistencia a comedor. Abarca los tres niveles de enseñanza (inicial, primaria y media), con un total general de casi mil alumnos de los cuales doscientos noventa y nueve pertenecen a Educación Primaria. 
Los grupos son numéricamente reducidos, con un promedio de veinte alumnos por clase.El local está ubicado en el barrio Pocitos, zona costera de la capital. El edificio, si bien es antiguo fue construido para ser usado como colegio, lo que implica que sus aulas y distribución fueron pensadas para tal fin. Está en permanente reciclaje y mantenimiento, lo que hace que sean tanto las aulas como los espacios destinados para otros fines, muy agradables. En locales ubicados en la misma manzana o enfrente, los alumnos transitan por las tres ramas de la enseñanza: Educación Inicial, Educación Primaria y Educación Media (incluyendo Bachillerato Internacional).

Este trabajo se acota, como ya explicamos, al área de español fundamentalmente, en el nivel primario ya que curricularmente es en este período en el cual se prevé la adquisición de la lectura y la escritura. Además el centrar la investigación en este nivel, que podría ser llamado intermedio, permite tener una visión de la incidencia de la Educación Inicial y la proyección de los conocimientos adquiridos para alcanzar las metas propuestas por la Educación Media.

En Educación Primaria, en el área de español, cumplen funciones catorce docentes de aula (14) de primer a sexto año. Además se tiene el apoyo de dos docentes encargados del Taller de Matemática, dos docentes en Taller de Lenguaje, en Biblioteca con otro docente y un bibliotecólogo, dos docentes de informática ( de $1^{\text {ero }}$ a $3^{\text {ero }}$. y de $4^{\text {to }}$. a $6^{\text {to }}$.), y dos docentes exclusivos para $5^{\text {to }}$ y $6^{\text {to }}$ año de electricidad y robótica, haciendo un total de 24 docentes que interactúan en el Nivel Primario. Se cuenta con instalaciones adecuadas para tales cometidos. El Taller de Informática cuenta con computadoras para cada alumno, equipadas con los últimos adelantos tecnológicos, como por ejemplo conexión a Internet, lo que brinda la posibilidad al alumnado de comunicarse con otras instituciones educativas, participar de video conferencias e intercambiar conocimientos. El Taller de Lenguaje funciona a cargo de dos docentes encargadas una de $2^{\mathrm{do}}$. y $5^{\text {to }}$. año y el resto de los grupos los atiende la otra docente. Las mismas concurren a los salones de clase y conjuntamente con el maestro a cargo realizan actividades previamente coordinadas.

La Biblioteca funciona durante todo el horario en un ámbito especialmente preparado para ello, ubicado, como lo indica el esquema adjunto en los anexos, en dos plantas. Los libros están al alcance de los alumnos y docentes. La bibliotecóloga y la maestra a cargo, siempre están a la orden para asesorar a los niños y adultos que lo requieran. Las clases de primaria concurren con su maestro una vez por semana (media hora) para realizar actividades que fomenten el gusto por la lectura y la escritura. El niño tiene la opción, en el recreo, de concurrir a biblioteca para leer, dibujar, mirar cuadros, etc.

\subsection{Instrumentos}

Se realizaron entrevistas semiestructuradas a los tres Directores de los niveles mencionados, tomándolos como informantes claves o calificados. Se realizaron pautas para ordenar dichas entrevistas y dar lugar a que cada informante pudiera expresarse lo más libremente posible. Las mismas fueron grabadas y realizadas conjuntamente por las dos investigadoras en el local escolar, con una duración aproximada de dos horas cada una.

A sugerencia de una de las informantes calificadas, entrevistamos a la Coordinadora de Lenguaje en Educación Media, ya que se consideró que era la persona que está más en contacto con el tema en ese nivel de enseñanza. A todos los docentes a cargo de las clases, a los talleristas de lenguaje e informática y a las personas encargadas de la biblioteca, se les entregaron cuestionarios abiertos con el fin de categorizarlos y concluir así el análisis cualitativo. Se encuestaron a todos los docentes de clase, de primero a sexto de primaria.

De igual manera, se encuestaron a los alumnos de primaria; el instrumento constó de preguntas que luego pudieran ser cuantificadas en sus resultado. De los doscientos noventa y nueve alumnos que hay en Enseñanza Primaria se eligieron al azar tres alumnos por clase, logrando así un muestreo cuotificado, tomando 36 alumnos que equivalen a un poco más del $10 \%$ de la población estudiada.

50 - Universidad ORT Uruguay 
El muestreo cuotificado se utiliza en poblaciones finitas. Con un tamaño muestral superior al 10\% (36) del tamaño de la población (299). Como criterio de cuotificación se utilizó el grado que estaba cursando el niño, con una asignación de igual número de casos para cada cuota, de forma tal que garantice una adecuada representación de la variabilidad total. Se utilizó el criterio de grado, en la medida que la edad es la variable que mejor determina la variabilidad de comportamiento.

\section{PROPUESTA DE ANÁLISIS DE DATOS}

En cuanto al análisis de datos se desgrabaron las entrevistas realizadas a las directoras para proceder a su análisis, y así obtener los objetivos y prioridades institucionales con respecto a la lectura $\mathrm{y}$ escritura.Con el formulario entregado a los docentes analizamos las respuestas en forma cualitativa, tomándolas una a una y estableciendo categorías.

Las categorías priorizadas fueron: concepto de lectura y escritura que el docente maneja, ubicación de estas actividades dentro de su proyecto educativo, incidencia de las actividades especiales en el curso (talleres, biblioteca), reconocimiento de estrategias que se llevan adelante, planificación y coordinación de las mismas.

De los cuestionarios a alumnos se ingresaron los datos a la computadora (Programa SURVEY) para analizar las diferentes variables, y se realizaron los cruzamientos para obtener datos más completos y poder realizar una conclusión más acabada.

\section{CONCLUSIONES FINALES}

A través de la investigación realizada podemos afirmar que el Centro Educativo es un ámbito propicio para el fomento de la lectura y la escritura. En él los alumnos disfrutan de estas actividades, las internalizan y la proyectan. La Institución en que se llevó a cabo la investigación está enmarcada en un ámbito social, económico y cultural muy bueno, brinda recursos para el fomento del aprendizaje, la comunidad que en ella habita tiene como prioridad la cultura.

Es vital el respaldo familiar que tenga el alumno. Como dice Smith (1994), la comunidad a la que ingresa desde su nacimiento es fundamental para que se inserte en un mundo culturalmente bueno. Pueden estar dadas o no las condiciones económicas y sociales, pero el objetivo primordial del núcleo familiar debe ser la cultura. Se saca en conclusión que si bien el medio educativo favorece o brinda buenas oportunidades para el aprendizaje, es notoriamente positivo que la familia y la comunidad en la que se inserte el niño brinden también el apoyo cultural necesario.

El contacto permanente con libros, mediante bibliotecas personales o familiares, y en la escuela proponiendo un lugar agradable donde estén los libros, hacen que los niños se acerquen a ellos con plena naturalidad, los dominen, los integren a su vida diaria. Es notorio en las respuestas dadas en la encuesta realizada a los alumnos que leer y escribir son asuntos disfrutables. En todas las actividades que se llevan adelante en la Institución se vislumbra el interés por comunicarse, trasmitir sus ideas, crear.

. La permanencia en las Direcciones y en los cargos docentes, hacen que el compromiso con un proyecto educativo sea mayor, permitiendo una continuidad en el proceso de enseñanza y aprendizaje. La coordinación y la posibilidad de encuentros entre docentes y con los Directores y Talleristas, permiten manejar un mismo idioma educativo, intercambio de ideas y enriquecimiento mutuo.

La Directora puede seleccionar su personal docente, lo que asegura un tácito acuerdo en la conducción educativa, una libre gestión del docente que siempre será consciente de cuál es el proyecto institucional. 
Del análisis se desprende que aunque no se cuente con espacios específicos para determinadas actividades, o con los recursos económicos adecuados, igual se puede incentivar el placer por leer y escribir, siempre que se enmarquen y surjan como necesidades vitales del niño.

Se preconiza la pedagogía centrada en el niño, cada uno debe ser artífice de su propio conocimiento, no obstante el docente y la institución educativa en su conjunto deben proporcionar los estímulos o disparadores necesarios para que sea más eficaz y redunde en menos esfuerzos.

Son la lectura y la escritura, dominadas ampliamente, las que permiten la entrada al mundo, logrando un individuo que maneje diferentes códigos e interactúe de diferentes formas con su medio ambiente. Permiten un ser independiente que logre por sí mismo un conocimiento cada vez más amplio, que sea crítico y reflexivo frente a cada información que se le brinda, investigando con creatividad y libertad. Es fundamental la Educación Inicial, no apuntando a enseñar algo preciso sino a "fomentar", como dice una de entrevistadas, las capacidades propias de cada individuo y a descubrirlas.

Es imperativo en este tiempo que el niño, desde el comienzo de su etapa educativa sienta estímulos, vea crecer sus posibilidades. La familia a su vez comienza allí a comprender, valorar y apoyar el trabajo docente, a "compartirlo", ya que el individuo que aprende es único y debe desarrollar sus potencialidades personales al máximo, en una tarea mancomunada.

En Educación Media se continúa este trabajo. El hecho de tener profesores específicos para cada asignatura, a veces hace poco posible la coordinación, o el apuntar a objetivos tan generales. Se trabaja en ello. La Institución, los docentes, las Direcciones, investigan sobre los problemas que se presentan y tratan de ir superándolos.

Las estrategias utilizadas fundamentalmente en primaria para fomentar la lectura y la escritura son los Talleres de Informática, Lenguaje y Biblioteca. La posibilidad de acercar al niño a conectarse con docentes y propuestas diferentes amplía su espectro de acción, dando la oportunidad de conocer distintas modalidades de trabajo, adaptándose a cada una de ellas.

Un buen conocimiento del grupo, reducido en número, logra que el docente domine la manera de acercarse y encarar el aprendizaje en cada individuo. En Biblioteca descubren el placer por leer, acercarse a las producciones de sus compañeros y valorarlas, respetando y exaltando las condiciones propias de cada uno.

Es un ámbito generador de estímulos, los niños lo utilizan con total libertad y alegría. Tiene la posibilidad de ser los artífices, exponiendo sus trabajos, objetando actividades, eligiendo los libros a adquirir.

Como se explica en la propuesta para 1998 dada por Biblioteca, se busca crear un ambiente acogedor, donde se escuche a los niños, se respeten sus lecturas y reflexiones silenciosas, marcando ellos mismos el ritmo.

El Taller de Informática da al alumno la posibilidad de manejar los adelantos tecnológicos, conocerlos, dominarlos, utilizándolos como una estrategia que agrada y atrae al mismo tiempo que logra cumplir con objetivos curricularmente propuestos.

El docente de clase es el regulador de todas las actividades y junto a sus colegas trata de perfilar una misma línea pedagógica, buscando mejorar los procesos de la enseñanza para facilitar el proceso de aprendizaje. El maestro, preparado técnicamente, con una buena formación docente, logra que en cada actividad planteada (geografía, historia, ciencias, etc.), el alumno use estas estrategias y perfeccione sus tareas al máximo.

Investigaciones anuales o periódicas facilitan al Director la continua evaluación del proceso de aprendizaje, posibilitándole, sobre la marcha, rever las estrategias didácticas aplicadas.

52 - Universidad ORT Uruguay 
De lo anteriormente expuesto surge que las estrategias institucionales son el apoyo en que sustentan las estrategias docentes; hay correlación y coherencia.

De los datos de la investigación queda claro que el alumno se siente partícipe de estas actividades, le interesan y opina sobre su marcha. Estas propuestas son motivadoras de la lectura y la escritura por placer. Toda la Institución se constituye en un ambiente muy propicio para la lectura y la escritura, aunque el análisis se focaliza, sobre todo, en este último aspecto.

Un docente aislado logra mucho menos que un grupo unido con un fin común claro, teniendo siempre como objetivo la superación del alumno y su inserción productiva y humana en el siglo que le espera. Cada estrategia debe convertirse para el individuo en algo transferible a otras situaciones, que permita la mejor adecuación y asimilación de los cambios. Lo más importante es aprender a aprender y el conocimientos más importante es el conocimiento de uno mismo.

\section{Bibliografía}

AIZPURO, A.; et. al. 1990. El escolar y la lectura. Montevideo, I.M.S.

ARELLANO, O. 1991. Avances de la Lectura en la Escuela Básica. En: Revista Lectura y Vida. 3er. Congreso Latinoamericano de Lectura $\mathrm{N}^{0} 1,: 46$ - 48, Bs. As., I.R.A.

BEST, J. 1974. Cómo investigar en Educación. Madrid, Morata.

BRASLAVSKY, B. 1992. La escuela puede. Bs. As., Aique.

CEPAL. 1990-91. Enseñanza Primaria y Ciclo de Educación Media en el Uruguay. Montevideo, CEPAL.

COOK, T. y REICHARDT, CH. 1986. Métodos Cualitativos y Cuantitativos en investigación educativa. Madrid, Morata.

DUBOIS, M. 1991. El proceso de la Lectura: de la Teoría a la Práctica. Bs. As., Aique.

DUBOIS, M. 1997. Educar en la lengua escrita, educar por la lengua escrita. En : Revista Lectura y Vida. $\mathrm{N}^{\circ} 2$,: pág. 39 - 44. Bs. As., I.R.A.

FERRÁ, C. 1993. Aprendizaje de Lecto-escritura. Montevideo, Amauta.

FERREIRO, E.; et. al. 1994. Nuevas perspectivas sobre los procesos de lectura y escritura. Lima, Siglo XXI.

FOX, D. 1981. El proceso de investigación en Educación. Pamplona, Universidad de Navarra.

FREEMAN, I.; SERRA, M. 1997. Alternativas positivas para la enseñanza tradicional de la Lectura. En: Revista Lectura y Vida, $\mathrm{N}^{\circ}$ 2,: 17 - 26. Bs. As., I.R.A.

HALLIDAY, M. 1973. Exploración de las funciones del lenguaje. Londres, Prentice Hall.

HARSTE, J. 1990. ¿Qué queremos significar ahora con Lectura?. En: Revista Lectura y Vida. N $^{\circ}$ 4,: pág. 5 - 10. Bs. As., I.R.A.

HUBERMAN, S. 1994. Cómo aprenden los que enseñan. Bs. As., Aique.

LACASA, P. 1994. Aprender en la escuela, aprender en la calle. Madrid, Visor.

NICOLETTI DE PRADA, M. 1993. Educación y medios de difusión: una postura diferente. Montevideo, Rosgal. 
POURTOIS, J.P. y HUGUETTE D. 1992. Epistemología e instrumentación en Ciencias Humanas. Barcelona, Herder.

POZNER, P. 1997. El directivo como gestor de aprendizajes escolares. Bs. As., Aique.

SMITH, F. 1994. De cómo la educación apostó al caballo equivocado. Bs. As., Aique.

SOLARI, A. 1991. La desigualdad educativa: problemas y críticas. En: Revista Anales Enseñanza Secundaria. Vol. 1. Montevideo, CODICEN.

STERN, S. 1994. Los desafíos de la equidad y la calidad en la Educación Primaria, una experiencia concreta: los C.R.A. Montevideo, Universidad Católica del Uruguay.

U.M.R.E. 1996. Informe del Proyecto MECAEP Evaluación 6tos. Años. Montevideo, ANEP BIRF.

WOOLFOLK, A. 1996. Psicología Educativa. .México D.F., Prentice Hall. 